\title{
Lensless Single Pixel Imaging with Laser Interference Patterns
}

\author{
Jarom Jackson $^{1}$, Dallin Durfee ${ }^{1}$
}

${ }^{1}$ Physics Department, Brigham Young University, Provo, UT, USA

We present proof of principle experiments for a single pixel [1] optical imaging method which should be capable of resolutions up to twice the normal Abbe diffraction limit (similar to other structured illumination techniques [2,3]), does not require any expensive high numerical aperture lenses, and does not need any optics to be placed close to the imaged object. This method, which we call Interference Pattern Structured Illumination Imaging (or IPSII), naturally combines high field of view with high resolution at low cost, making it potentially well suited for low cost medical or scientific analysis instruments. Because it requires only a single pixel detector, it may also be well suited to imaging in extreme wavelength regimes (such as ultraviolet, $\mathrm{x}$-ray or the far infrared) where lenses or highresolution imaging arrays are difficult to manufacture.

The imaging process of IPSII, similar to magnetic resonance imaging (MRI), directly measures components of the objects spatial Fourier transform. This is done by illuminating the object with 2D sinusoidal patterns, while measuring the reflectance of, or transmittance through the object with a single pixel detector such as a photodiode or photomultiplier tube. The resulting signal is directly related to the Fourier transform of the object evaluated at the k-vector of the illuminating pattern. By scanning the kvector in 2D, the object's Fourier transform can be mapped out. The object image is then reconstructed by performing an inverse transform on the data, with the resolution of the reconstruction proportional to the minimum spacing (maximum k-vector) of the illumination patterns.

We generate the illumination patterns by interfering two collimated laser beams. This results in a sinusoidal pattern whose fringe spacing depends on the wavelength of the lasers and the angle between them, but does not depend on the NA of any of the laser optics, or the distance of the object from the optics. Scanning the wavenumber is done simply by scanning the angle between the two beams. For each k-vector, we also scan the phase of the pattern by introducing a slight frequency difference between the two beams. The resulting detector signal $s$ is sinusoidal, with the amplitude and phase giving the complex value of a spatial Fourier component. The complex representation of $s$, as a function of the 2D wavevector components $k_{x}$ and $k_{y}$ is given by,

$$
s\left(k_{x}, k_{y}\right)=\iint_{k_{x}, k_{y}} m(x, y) A\left(x, y ; k_{x}, k_{y}\right) e^{i\left(k_{x} x+k_{y} y\right)}
$$

where $m(x, y)$ is the transmission (or reflectance) of the object and $A\left(x, y ; k_{x}, k_{y}\right)$ is the overlap of the two beam profiles. The overlap function contains any mode irregularities or phase distortions and is a function of the wavevector because the beam angle affects the projection of the laser mode onto the object. It is also approximately constant if the laser mode is high quality and larger than the object, in which case the signal $s$ is proportional to the Fourier transform of $m(x, y)$, evaluated at $k_{x}$ and $k_{y}$.

We have conducted simple 1D, low resolution experiments to demonstrate the feasibility of IPSII in practice, and develop methods to deal with a non-ideal overlap function arising from imperfections in mirrors and other laser optics, or in the laser mode itself. Our imaging device (shown in Figure 1) consists of a Mach-Zehnder interferometer. The frequency difference is generated by ramping a piezo 
mounted mirror, while the angle between the interfering beams is scanned with a single motor actuated mirror axis. A photodiode mounted to a pinhole placed in one output arm of the interferometer serves as a phase reference. The object (one or two wires) is placed in the second output arm, with photodiodes behind and in front to detect the transmission and reflectance.

While this initial simplified implementation was limited in resolution, we've shown that the principles behind IPSII can be put into practice. Figure 1 shows one such imaging test in which the transmission signal through two wires is shown, as well as the beam profile of the laser (which was imaged in the same setup by removing the object). In a second experiment (see Figure 2), the shadow of a single wire was imaged using an imperfect laser mode. The phase was further distorted by placing a microscope slide in one arm of the interferometer. This experiment demonstrates that beam wavefront phase distortion only affects the complex phase of the image reconstruction, which can be neglected if both positive and negative $\mathrm{k}$ values are measured (by scanning through positive and negative angles).

We are currently working on a next generation device capable of 2D imaging and higher resolution.

References:

[1] M G L Gustafsson, Journal of Microscopy 198 (2000), p. 82-87.

[2] R Horisaki, H Matsui and J Tanida, Applied optics 56.14 (2017), p. 4085-4089.

[3] L M Hirvonen et al, European Biophysics Journal 38(6) (2009), p. 807-12.
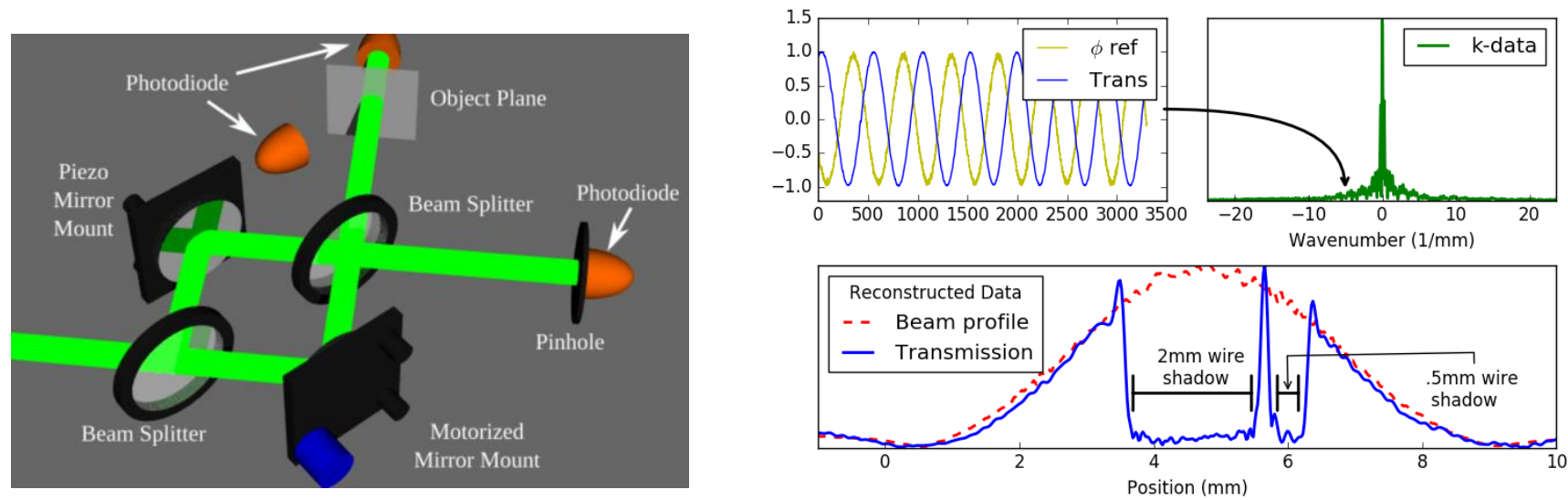

Figure 1. Left: Schematic diagram of IPSII experiment. Right: 1D low resolution proof of principle imaging of a pair of wires, showing the raw signal data from the transmission detector and reference pinhole, the measured spatial spectrum, and a reconstruction of the object shadow and the beam profile.
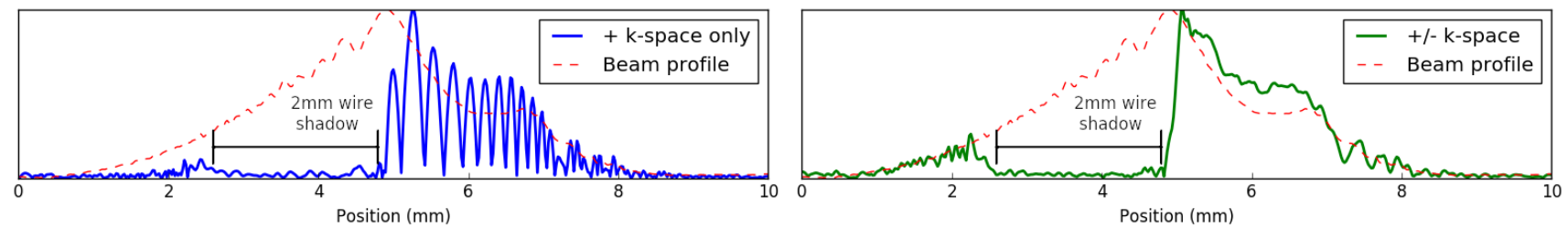

Figure 2. 1D shadow of a single wire imaged with a bad mode and strongly distorted wavefronts. Left: image reconstruction using only positive k-values. Right: image reconstruction using positive and negative k-values, and eliminating phase distortion effects by discarding the complex phase of the reconstruction. 